\title{
Use of the Vsoc-maze to Study Sociability and Preference for Social Novelty in Rodents \\ Sara Martínez-Torres ${ }^{\#}$, Maria Gomis-González ${ }^{\#, \$ a, ~ A l b a ~ N a v a r r o-R o m e r o ~}{ }^{\$ b}$, Rafael Maldonado and Andrés Ozaita*
}

Laboratory of Neuropharmacology. Department of Experimental and Health Sciences, University

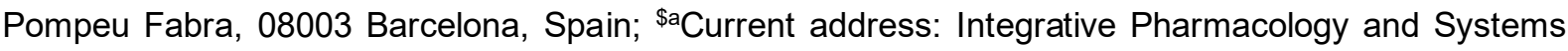
Neuroscience Research Group, Hospital del Mar Medical Research Institute, 08003 Barcelona, Spain; ${ }^{\$ b}$ Current address: Neurodegenerative Diseases Research Group, Vall dHebron Research Institute (VHIR)-Network Center for Biomedical Research in Neurodegenerative Diseases (CIBERNED)Autonomous University of Barcelona, 08035 Barcelona, Spain

*For correspondence: andres.ozaita@upf.edu

\#Contributed equally to this work

\begin{abstract}
[Abstract] Studying social behavior in mouse models empowers the understanding of the neurobiological mechanisms involved, which are affected in neuropsychiatric disorders, allowing the evaluation of therapeutic strategies. Behavioral methods available are time-consuming and reducing the length of behavioral sessions may render more manageable experiments and reduce animal stress. We validated a new reliable and sensitive method to study two features of social behavior (sociability and preference for social novelty) in two strains of male mice, the C57BL/6J inbreed strain and the CD1 (ICR) outbreed strain, using a modified version of the V-shaped maze (Vsoc-maze). The Vsoc-maze for sociability and preference for social novelty improves time performance by shortening the length of the sessions, and reduces variability compared to the classical approach performed in the three-chamber apparatus. Altogether, the Vsoc-maze allows evaluating the specific alterations of social behavior in mice in a time-efficient and reproducible manner.
\end{abstract}

Keywords: V-maze, Social behavior, Mouse model, Sociability, Preference for social novelty

[Background] Social behaviors are important in numerous species to establish the networks and relationships that define social communities (Berry and Bronson, 1992). Among those behaviors, sociability is defined as the tendency to seek out social interaction (Caldwell, 2012). Some neuropsychiatric disorders, such as autism spectrum disorders (ASD) display a marked alteration in sociability combined with other features (American Psychiatric Association, 2013). This characteristic highlights the need for experimental behavioral settings in animal models to address the research on such complex multi-facetted disorders (Caldwell, 2012). Mus musculus is a social specie showing reciprocal social interactions, sexual and parenting behaviors, territorial scent marking and aggressive behaviors (Ricceri et al., 2007; Silverman et al., 2010). The wide repertoire of mouse behaviors makes this specie suitable for modeling human disorders characterized by disruptions in social recognition and social behavior (Crawley, 2004; Yang et al., 2007). Such rodent models warrant the evaluation of potential therapeutic approaches for treatment (Moy et al., 2004). Commonly, mice conserve a 
characteristic pattern of social behavior, initiating social contact and approach when exposed to an unfamiliar conspecific (Moy et al., 2004).

Several paradigms have been described to measure social behavior in mouse models (Silverman et al., 2010). Among those, the most common is the Crawley's sociability test also called three-chamber apparatus (Chadman et al., 2008; McFarlane et al., 2008; Moy et al., 2008), which allows to study two different parameters: sociability and preference for social novelty. Sociability is defined as the tendency of the experimental mouse to spend more time exploring an unfamiliar mouse than exploring an object or an empty chamber. Instead, preference for social novelty is defined as the propensity to spend more time exploring a new unfamiliar mouse than the now-familiar mouse that was explored in the sociability phase (Moy et al., 2004; Yang et al., 2011). Due to the length of the different phases in the Crawley's sociability test, the procedure is time-consuming and experimental variability is sometimes troublesome.

We have adapted a V-maze, previously used successfully to evaluate novel object-recognition memory (Puighermanal et al., 2009; Busquets-Garcia et al., 2011 and 2013; Navarro-Romero et al., 2019), to assess social behavior. We found that the procedure using this new maze (Vsoc-maze) provides an advantageous approach in terms of time, while maintaining accuracy, to reveal particular social phenotypes in two mouse lines frequently used in biomedical research.

\section{Materials and Reagents}

1. Tissue paper

2. Experimental mice (e.g., C57BL/6J and CD1 male mice, 12-16 weeks old, Charles River Laboratory)

3. Stranger mice (STR mice) (e.g., juvenile 3-4 weeks old (postnatal day 21 to 28) male C57BL/6J mice, Charles River Laboratory)

4. $30 \%$ ethanol $(v / v)$

\section{Equipment}

1. Vsoc-maze

We used a modified version of the V-shaped maze (Busquets-Garcia et al., 2011) (Vsoc-maze, Figure 1). It consists of two structures: the maze wall (150 mm high), made of black Plexiglas (6 $\mathrm{mm}$ width) and the maze lid, made of transparent Plexiglas. Corridors in the Vsoc-maze are $300 \mathrm{~mm}$ long and $45 \mathrm{~mm}$ wide (internal measures). Two small chambers (82 $\mathrm{mm}$ long) were created at the end of the corridors when the lid was inserted into the Vsoc-maze. These chambers were used to allocate the juvenile stranger mice. The design was deliberately simple to enhance the exploratory activity of the experimental mouse under analysis, and to facilitate the handling of the animals and the cleaning between sessions.

2. Three-chamber apparatus 
It consisted in a rectangular box made of Plexiglas $(405 \mathrm{~mm}$ wide $\times 600 \mathrm{~mm}$ long $\times 150 \mathrm{~mm}$ high) divided in three-identical-chambers ( $405 \mathrm{~mm}$ wide $\times 200 \mathrm{~mm}$ long $\times 150 \mathrm{~mm}$ high) by two Plexiglas walls containing small openings, which measure $100 \mathrm{~mm}$ wide $\times 50 \mathrm{~mm}$ high, to allow mouse access between chambers as previously described (Kaidanovich-Beilin et al., 2011). The juvenile stranger mice were enclosed in a round wire cage in the side chambers. The wire cage was $110 \mathrm{~mm}$ high $\times 105 \mathrm{~mm}$ diameter and had vertical bars spaced $10 \mathrm{~mm}$, which allow sniffing and exploration. There was a weighted cup on top of the wire cage to prevent the experimental animal from climbing.

3. Video Camera (e.g., Sony Handycam camcorder, HDR-CX240)

4. Stopwatches (x2) (e.g., Sper Scientific, 810029)

A

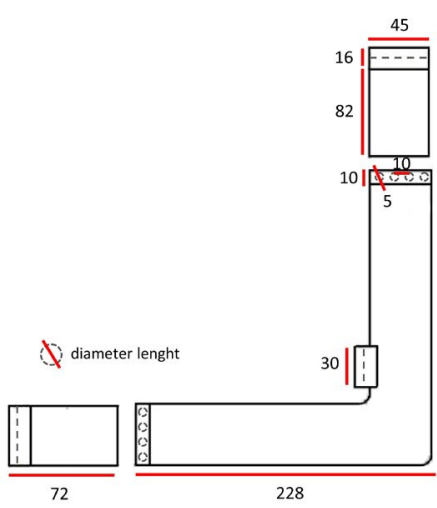

C

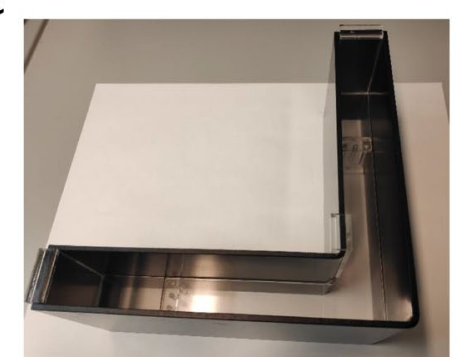

B

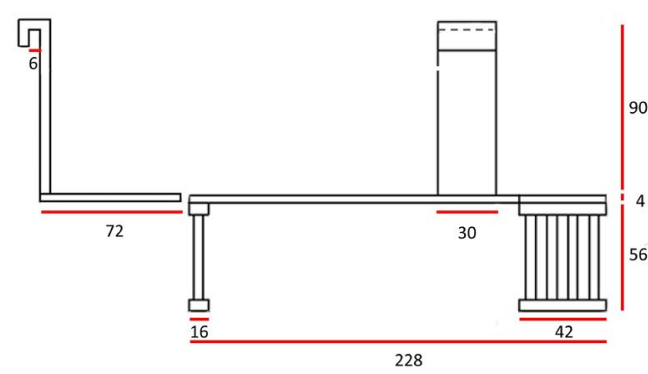

D

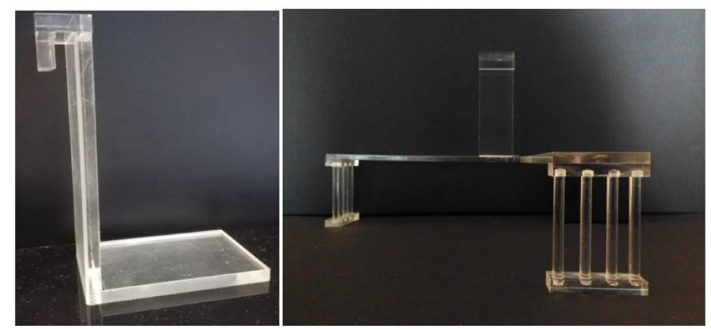

Figure 1. Design of the Vsoc-maze. Top view ( $A$ and $C$ ) and side view ( $B$ and $D$ ) of the Vsoc-maze and the Plexiglas transparent lid showing its dimensions (in $\mathrm{mm}$ ) used in Martínez-Navarro et al. (2019). Photographs show the Vsoc-maze with the transparent lid (C), and a detail of the lid which includes the Plexiglas bars for mice to interact (D). 


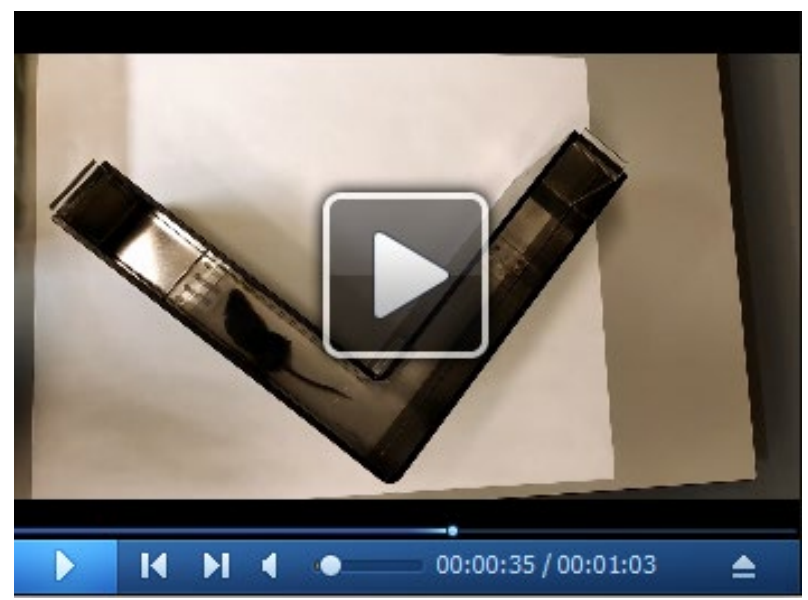

Video 1. Vsoc-maze set-up and mouse handling for the test. (All animal procedures were conducted following ARRIVE (Animals in Research: Reporting In Vivo Experiments) (Kilkenny et al., 2010) guidelines and standard ethical guidelines (European Communities Directive 2010/63/EU) and approved by the local ethical committee (Comitè Ėtic dExperimentació Animal-Parc de Recerca Biomèdica de Barcelona, CEEA-PRBB).)

\section{Software}

1. STATISTICA (StatSoft) software, used to performed the statistical analysis

2. GraphPad Prism software (GraphPad Software, USA), used for artwork

\section{Procedure}

The mazes (Vsoc-maze and three-chamber apparatus) were used in a sound-attenuated room with dim illumination 4-7 lux. A digital camera on top of the maze was used to record the sessions. On-line image in a contiguous room was available to the observer through a close-circuit camera situated on top of the maze. All three phases of the social test (habituation, sociability and preference for social novelty) were performed consecutively (Figure 2). Vsoc-maze phases lasted $5 \mathrm{~min}$ as it has been described that during the first five minutes of interaction the majority of the social behaviors take place (Nadler et al., 2004). The sessions in the three-chamber apparatus usually lasts $10 \mathrm{~min}$ (Kaidanovich-Beilin et al., 2011). For comparison purpose, exploratory behavior in the three-chamber apparatus was computed at 5 min (Figure 3) and $10 \mathrm{~min}$ (Figure 4).

All mice were housed four per cage and maintained in standard environment conditions of temperature $\left(21{ }^{\circ} \mathrm{C} \pm 1{ }^{\circ} \mathrm{C}\right)$ and humidity $(55 \% \pm 10 \%)$ with food and water ad libitum. All the experiments were performed during the light phase of the dark/light cycle (lights on at 8 a.m. and off at 8 p.m.). Before starting the experiment, mice were habituated in the experimental room and handled for 1 week. 
Phase I. Habituation

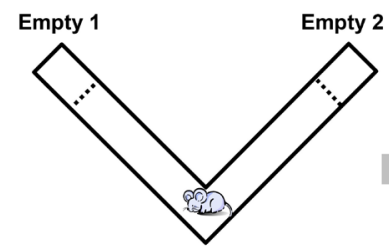

Phase II. Sociability

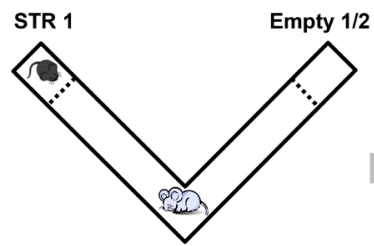

Phase III. Preference for social novelty

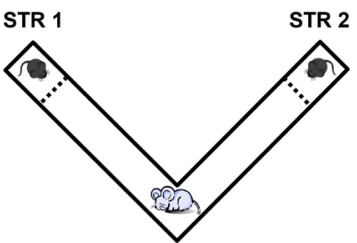

Figure 2. Scheme of the sociability and preference for social novelty test (Martínez-Navarro et al., 2019). There are three different phases: Phase I (habituation), Phase II (sociability) and Phase III (preference for social novelty). Each phase lasts $5 \mathrm{~min}$. During this time the exploration of the experimental mouse towards the chambers at the end of the corridors was recorded on the three phases: both chambers empty (Phase I), stranger-1 (STR 1) vs. empty chamber (Phase II) and stranger-1 (STR 1) vs. stranger-2 (STR 2) (Phase III).

A. Habituation session (Phase I)

The habituation session allows mice to freely explore and familiarize with the Vsoc-maze.

1. To record the whole experiment, turn on the video recording system.

2. Place the experimental mouse by lifting the Vsoc-maze, with the chambers empty, at the starting point (crossing-point between both arms).

3. Let the experimental mouse explore the maze for $5 \mathrm{~min}$.

4. During this time, use two stopwatches and assign each stopwatch to count the exploration time of each chamber. Then, measure the exploration time for each chamber through the image obtained by the closed-circuit camera.

5. At the end of the session, record the time exploring each empty chamber.

Notes:

a. It is not necessary to turn off the video recording and/or the data acquisition system. There is no need to move the experimental mouse from the Vsoc-maze.

b. Although in our experience it would not be necessary, an inter-trial period could be included in which the experimental mouse is removed from the Vsoc-maze after habituation. During the inter-trial period the stranger 1 (STR 1) is placed in the chamber (see Sociability session, Phase II). Performing this inter-trial period may avoid experimenter's manipulation of the maze while the experimental mouse is inside the Vsoc-maze. Moreover, it would allow cleaning any urine or defecation in the Vsoc-maze which may affect the exploratory behavior of the experimental mouse during the next Phases of the procedure.

B. Sociability session (Phase II)

The sociability session is performed just after the habituation session.

1. A juvenile mouse assigned as (STR 1) is placed in one of the small chambers located at the end of one of the corridors (both corridors were alternated during the experiments). The mouse 
is placed in the chamber from the top of the maze, before placing the lid of the chamber.

2. The sociability session lasts $5 \mathrm{~min}$.

3. Use the stopwatches to count the time that the experimental mouse spends exploring each chamber. A preference for spending more time exploring the chamber with the STR 1 than the empty chamber should be obvious. This juvenile mouse was used as STR 1 for all the experiment.

4. At the end of the sociability session, record the exploration times for both chambers. Maintain the experimental mouse and STR 1 in the Vsoc-maze and start the last phase of the test. Notes:

a. An inter-trial period between Phase II and III could be included, where the experimental mouse is removed from the Vsoc-maze.

b. Alternatively, the STR 1 may be changed to the previously empty chamber before introducing STR 2 in the chamber previously occupied by STR 1. Under these conditions the experimenter can be confident that the exploration time devoted to STR 1 and STR 2 in the next Phase is due to a social novelty preference rather than spatial novelty (new STR 2 in previously empty chamber).

c. Additionally, Phase II may be extended beyond $5 \mathrm{~min}$ in the case that social habituation-dishabituation behavior may be studied. Experimental mice may reduce the interest for exploring STR 1 as the Phase II progresses, as shown elsewhere (Hitti and Siegelbaum, 2014). By running Phase II for longer, a decay in exploration as a function of session time can be revealed.

C. Preference for social novelty session (Phase III)

The preference for social novelty session is performed just after the sociability session.

1. The test session lasts $5 \mathrm{~min}$.

2. A second novel mouse assigned as stranger 2 (STR 2) is placed from the top inside the previously empty chamber, while the STR 1 remains inside the same chamber. During test time the experimental mouse is allowed to explore all the apparatus. This juvenile mouse was used as STR 2 for all the experiment.

3. Use the stopwatches to count the time that the experimental mouse spends exploring each chamber. A preference for spending more time exploring the chamber with the STR 2 , which is the novel mouse, than the STR 1 , the familiar mouse should be obvious.

D. Post-test cleaning session

1. Remove the experimental mouse and the stranger mice from the apparatus by lifting the whole maze, and return mice to their home cage.

2. Clean the maze with $30 \%(\mathrm{vol} / \mathrm{vol})$ ethanol to avoid olfactory cues.

3. Do the test with another animal if it is necessary (back to step 2 in Habituation session [Phase I]). 
4. When the data acquisition for the Whole experiment is finished, export the experimental data and analyze the results (see Data analysis).

Note: We compared the exploration times in both settings, the Vsoc-maze and the three-chamber apparatus, using an inbreed and an outbreed mouse strain (C57BL/6J and CD1 mice, respectively). In order to compare time efficiency between mazes, both strains were analyzed for $5 \mathrm{~min}$ in the Vsoc-maze (Figure 3) and for $5 \mathrm{~min}$ (Figure 3) and $10 \mathrm{~min}$ (Figure 4) in the three-chamber maze. The Vsoc-maze setting provided quite similar results in the habituation session (Phase I) for both strains (Figures $3 A$ and $3 B$ ). These results were variable for CD1 mice in the three-chamber maze (Figure $3 B$ ). In the sociability phase (Phase II), both mouse strains demonstrated a significant preference $(P<0.001)$ for the chamber containing the juvenile STR 1 compared to the empty chamber. Such preference was evident in the 5 min period in both mazes (Figures $3 C$ and $3 D$ ) and in the 10 min for the three-chamber apparatus (Figure 4). Finally, on the $5 \mathrm{~min}$ session to assess preference for social novelty (Phase III), only mice analyzed in the Vsoc-maze, independent of their strain, showed a significant predilection $(P<$ 0.001) for exploring the new-unfamiliar juvenile mouse (STR 2) compared to the now-familiar mouse (STR 1) (Figures $3 E$ and $3 F$ and Figure 4). C57BL/6J mice took longer to show a clear preference for social novelty in the three-chamber maze since such preference was only apparent after 10 min (Figure $3 E$ and Figure 4A). Alternatively results can be represented using a social index (Figure 5).

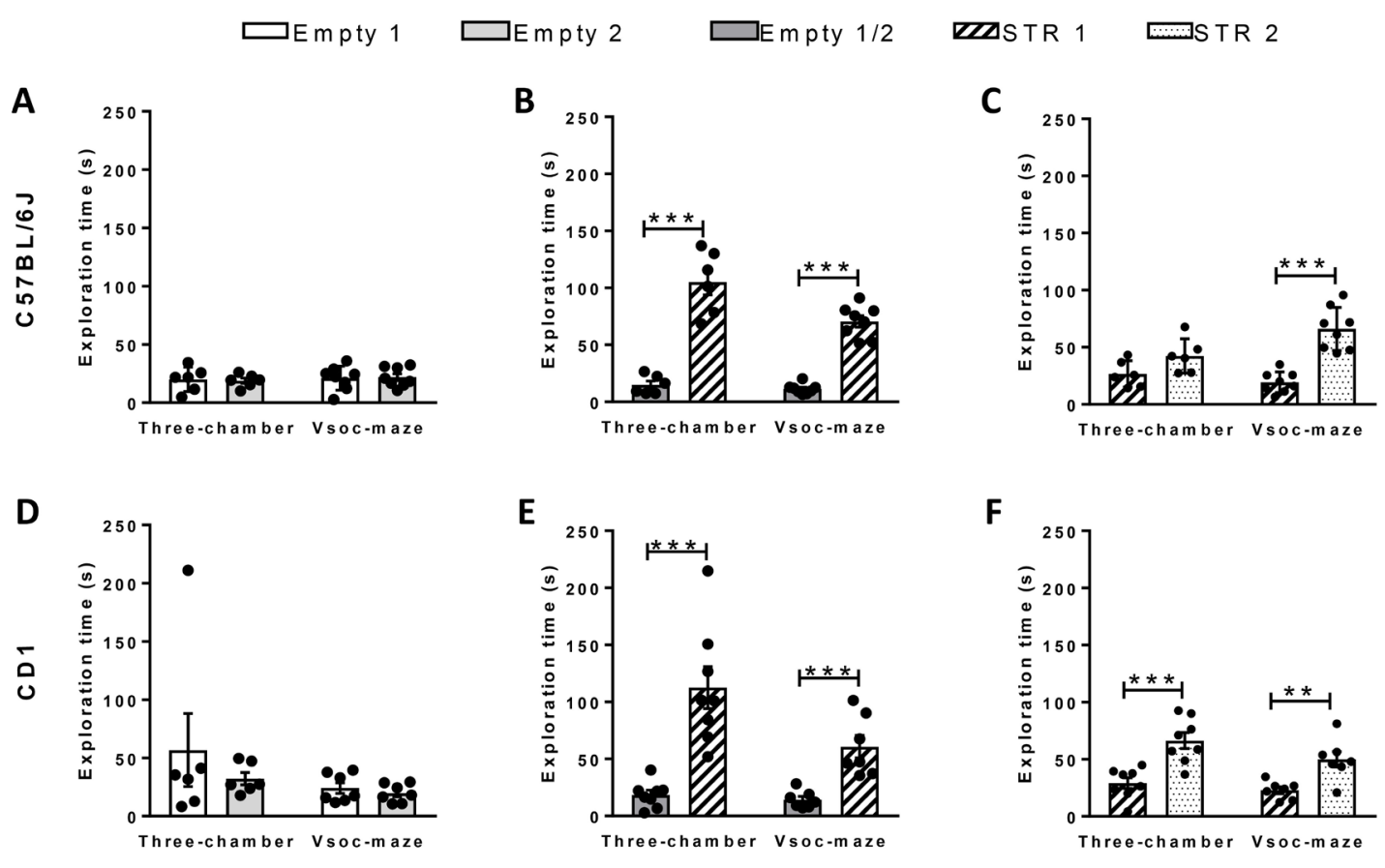

Figure 3. Comparison of social behavior of two mouse lines using the three-chamber apparatus and the Vsoc-maze. Exploration time of C57BL/6J (A-C) and CD1 (D-F) mice during the three phases of the experiment. Time exploring the empty chambers during phase I (A and D). Time spent exploring either the empty chamber or STR 1 during phase II (B and E). Time spent exploring either STR 1 or STR 2 during phase III (C and F). Three-chamber 
C57BL/6J, $n=6$; CD1, $n=7$; Vsoc-maze C57BL/6J, $n=8 ; C D 1, n=8$ ). Data are expressed as mean \pm s.e.m. Statistical significance was calculated by Student's $t$-test. ${ }^{\star} P<0.05$; ${ }^{*} P<0.01$; ${ }^{* * *} P<0.001$ (chamber comparison).
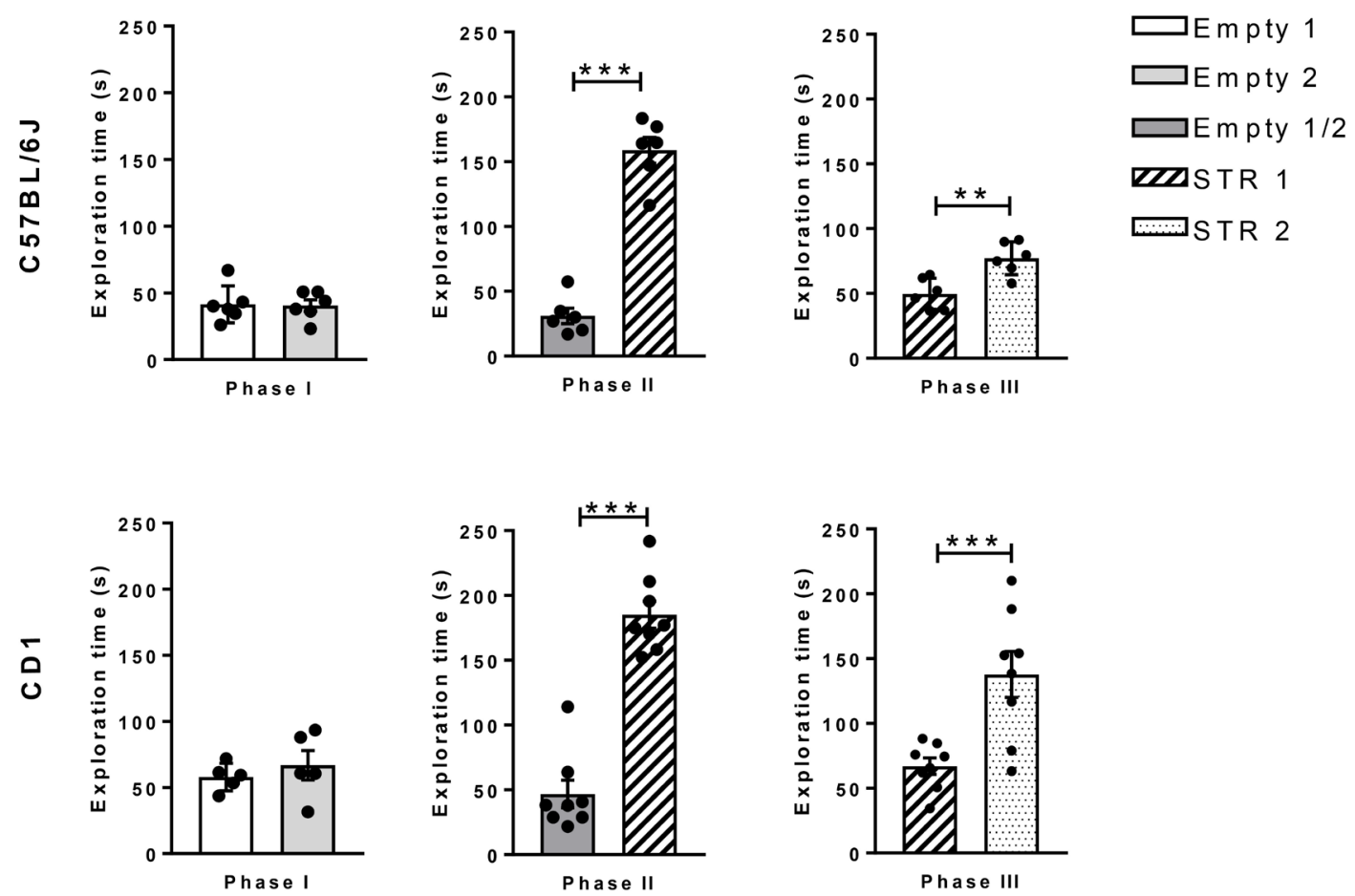

Figure 4. Total exploration time performing the three-chamber social test for $10 \mathrm{~min}$. Exploratory behavior of C57BL/6J mice (A) and CD1 mice (B) in the three-chamber social test during the $10 \mathrm{~min}$ of each phase. Data are expressed as mean \pm S.E.M. Statistical significance was calculated by Student's $t$-test. ${ }^{* *} P<0.01 ;{ }^{* * *} P<0.001$ (chamber comparison). 

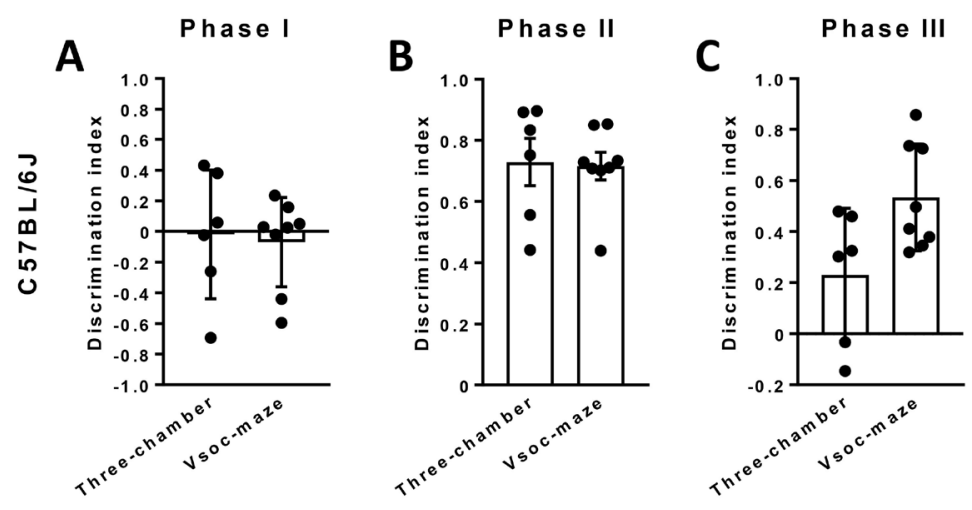

D

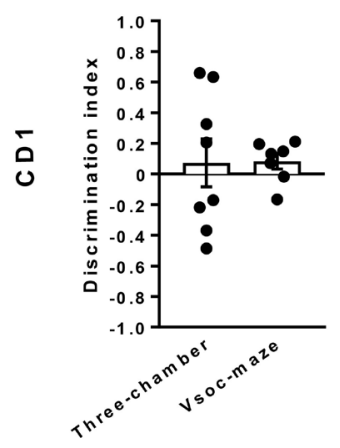

E

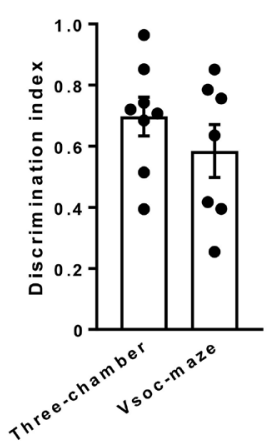

$\mathbf{F}$

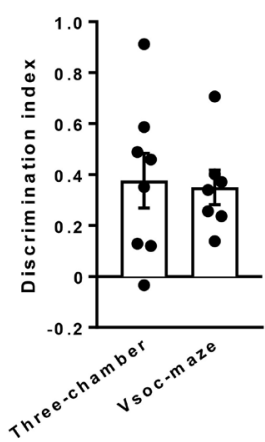

Figure 5. Discrimination index of C57BL/6J and CD1 mice during the three phases of the experiment. Discrimination index of the empty chambers during phase I (A and D). Discrimination index between the empty chamber or STR 1 during phase II ( $B$ and $E$ ). Discrimination index between STR1 or STR 2 during phase II (C and F). Three-chamber C57BL/6J, $n=6$; CD1, $n=7$; Vsoc-maze C57BL/6J, $n=8$; CD1, $n=8$ ). Discrimination index was calculated as the difference between the time spent exploring the novel stimulus minus the time exploring the familiar stimulus divided by the total exploration time. Data are expressed as mean \pm s.e.m.

\section{Data analysis}

1. Exploration time is considered when the experimental mouse, with all four paws in the floor, directs the nose in close proximity $(1 \mathrm{~cm})$ to the vertical bars at the end of the corridors (Yang et al., 2011). Sniffing while climbing was not counted as exploration time.

2. In each phase the time exploring both chambers (whether they are empty or they hold a juvenile stranger mouse) is computed.

3. Animals that explore the chamber or stranger mouse $<3 \mathrm{~s}$ during any 5 min session are excluded from subsequent analysis.

4. The mean time spent exploring both chambers were used to perform Student's $t$-test analysis. All results are expressed as mean \pm S.E.M. and comparisons are considered statistically 
significant when the level of significance is less than 0.05 . The statistical analysis is performed using STATISTICA (StatSoft) software.

\section{Notes}

1. An animal does not explore the chamber or stranger mouse presenting a very low total exploration time $(<3 \mathrm{~s})$. There can be different reasons:

a. The animal does not explore the maze during the habituation session: Exclude this mouse from the analysis.

b. The stranger mouse is not adequate for the test, for example because it presents hyperactive behavior. In this case, the stranger mouse is not considered for the experiment and it is replaced for another.

c. After a pharmacological treatment or a genetic manipulation, the mouse does not explore the maze. Probably, the treatment or the genetic background alters locomotion activity. You may change the timing of the treatment or use another test.

2. Control animals do not show good social behavior and prefer to explore one of the stranger mice. A solution can be to consider whether changing the stranger mouse is convenient.

3. Results are not reproducible. It can be due to the presence of odor cues or any other type of stressor present in the experimental room. The maze must be correctly cleaned between each animal exposure and the experimental conditions during the test must be controlled.

\section{Acknowledgments}

This work was supported by the Spanish Ministerio de Economía, Innovación y Competitividad (MINECO) [\#BFU2015-68568-P to A.O., \#SAF2017-84060-R-AEI/FEDER-UE to R.M.]; European Commission [NeuroPain, \#FP7-HEALTH-F2-2013-602891 to R.M.]; Spanish Instituto de Salud Carlos III [\#RD16/0017/0020 to R.M.]; Spanish "Plan Nacional Sobre Drogas" of the Spanish Ministerio de Salud [\#PNSD-20171068 to R.M.]; the Generalitat de Catalunya [2017SGR-669 to R.M., ICREA Acadèmia2015 to R.M., ICREA Acadèmia2016 to A.O.]; Grant "Unidad de Excelencia María de Maeztu", funded by the MINECO [\#MDM-2014-0370]; PLAN E (Spanish Plan Español para el Estímulo de la Economía y el Empleo); and FEDER funding is also acknowledged.

S.M-T. is a recipient of a predoctoral fellowship from Generalitat de Catalunya, Spain [FI-B00531 2016]. M.G-G. was supported by a predoctoral fellowship from FRAXA Research Foundation. A.N-R was supported by a predoctoral fellowship (Spanish Ministerio de Educación y Cultura).

We thank Francisco Porrón and Dulce Real for expert technical assistance and the Laboratory of Neuropharmacology-NeuroPhar for helpful discussion.

The methodology described was previously used in Martínez-Navarro et al. (2019). 


\section{Competing interests}

Authors declare no conflict of interest.

\section{Ethics}

All animal procedures were conducted following ARRIVE (Animals in Research: Reporting In Vivo Experiments) (Kilkenny et al., 2010) guidelines and standard ethical guidelines (European Communities Directive 2010/63/EU) and approved by the local ethical committee (Comitè Ėtic dExperimentació Animal -Parc de Recerca Biomèdica de Barcelona, CEEA-PRBB).

\section{$\underline{\text { References }}$}

1. American Psychiatric Association (2013) Diagnostic and Statistical Manual of Mental Disorders. 5th Edition, APA, Washington, DC.

2. Berry, R. J. and Bronson, F. H. (1992). Life history and bioeconomy of the house mouse. Biol Rev Camb Philos Soc 67(4): 519-550.

3. Busquets-Garcia, A., Puighermanal, E., Pastor, A., de la Torre, R., Maldonado, R. and Ozaita, A. (2011). Differential role of anandamide and 2-arachidonoylglycerol in memory and anxiety-like responses. Biol Psychiatry 70(5): 479-486.

4. Busquets-Garcia, A., Gomis-Gonzalez, M., Guegan, T., Agustin-Pavon, C., Pastor, A., Mato, S., Perez-Samartin, A., Matute, C., de la Torre, R., Dierssen, M., Maldonado, R. and Ozaita, A. (2013). Targeting the endocannabinoid system in the treatment of fragile $X$ syndrome. Nat Med 19(5): 603-607.

5. Caldwell, H. K. (2012). Neurobiology of sociability. Adv Exp Med Biol 739: 187-205.

6. Chadman, K. K., Gong, S., Scattoni, M. L., Boltuck, S. E., Gandhy, S. U., Heintz, N. and Crawley, J. N. (2008). Minimal aberrant behavioral phenotypes of neuroligin-3 R451C knockin mice. Autism Res 1(3): 147-158.

7. Crawley, J. N. (2004). Designing mouse behavioral tasks relevant to autistic-like behaviors. Ment Retard Dev Disabil Res Rev 10(4): 248-258.

8. Hitti, F. L. and Siegelbaum, S. A. (2014). The hippocampal CA2 region is essential for social memory. Nature 508(7494): 88-92.

9. Kaidanovich-Beilin, O., Lipina, T., Vukobradovic, I., Roder, J. and Woodgett, J. R. (2011). Assessment of social interaction behaviors. $J$ Vis $\operatorname{Exp}(48)$.

10. Kilkenny, C., Browne, W., Cuthill, I. C., Emerson, M., Altman, D. G. and Group, N. C. R. R. G. W. (2010). Animal research: reporting in vivo experiments: the ARRIVE guidelines. $\mathrm{Br} \mathrm{J}$ Pharmacol 160(7): 1577-1579.

11. Martínez-Navarro, M., Lara-Mayorga, I. M., Negrete, R., Bilecki, W., Wawrzczak-Bargieła, A., Gonçalves, L., Dickenson, A.H., Przewłocki, R., Baños, J.E., Maldonado R. (2019). Influence of 
behavioral traits in the inter-individual variability of nociceptive, emotional and cognitive manifestations of neuropathic pain. Neuropharmacology 148:291-304.

12. McFarlane, H. G., Kusek, G. K., Yang, M., Phoenix, J. L., Bolivar, V. J. and Crawley, J. N. (2008). Autism-like behavioral phenotypes in BTBR T+tf/J mice. Genes Brain Behav 7(2): 152-163.

13. Moy, S. S., Nadler, J. J., Perez, A., Barbaro, R. P., Johns, J. M., Magnuson, T. R., Piven, J. and Crawley, J. N. (2004). Sociability and preference for social novelty in five inbred strains: an approach to assess autistic-like behavior in mice. Genes Brain Behav 3(5): 287-302.

14. Moy, S. S., Nadler, J. J., Young, N. B., Nonneman, R. J., Segall, S. K., Andrade, G. M., Crawley, J. N. and Magnuson, T. R. (2008). Social approach and repetitive behavior in eleven inbred mouse strains. Behav Brain Res 191(1): 118-129.

15. Nadler, J. J., Moy, S. S., Dold, G., Trang, D., Simmons, N., Perez, A., Young, N. B., Barbaro, R. P., Piven, J., Magnuson, T. R. and Crawley, J. N. (2004). Automated apparatus for quantitation of social approach behaviors in mice. Genes Brain Behav 3(5): 303-314.

16. Navarro-Romero, A., Vazquez-Oliver, A., Gomis-Gonzalez, M., Garzon-Montesinos, C., Falcon-Moya, R., Pastor, A., Martin-Garcia, E., Pizarro, N., Busquets-Garcia, A., Revest, J. M., Piazza, P. V., Bosch, F., Dierssen, M., de la Torre, R., Rodriguez-Moreno, A., Maldonado, R. and Ozaita, A. (2019). Cannabinoid type-1 receptor blockade restores neurological phenotypes in two models for Down syndrome. Neurobiol Dis 125: 92-106.

17. Puighermanal, E., Marsicano, G., Busquets-Garcia, A., Lutz, B., Maldonado, R. and Ozaita, A. (2009). Cannabinoid modulation of hippocampal long-term memory is mediated by mTOR signaling. Nat Neurosci 12(9): 1152-1158.

18. Ricceri, L., Moles, A. and Crawley, J. (2007). Behavioral phenotyping of mouse models of neurodevelopmental disorders: relevant social behavior patterns across the life span. Behav Brain Res 176(1): 40-52.

19. Silverman, J. L., Yang, M., Lord, C. and Crawley, J. N. (2010). Behavioural phenotyping assays for mouse models of autism. Nat Rev Neurosci 11(7): 490-502.

20. Yang, M., Scattoni, M. L., Zhodzishsky, V., Chen, T., Caldwell, H., Young, W. S., McFarlane, H. G. and Crawley, J. N. (2007). Social approach behaviors are similar on conventional versus reverse lighting cycles, and in replications across cohorts, in BTBR $T+t f / J, C 57 B L / 6 J$, and vasopressin receptor 1B mutant mice. Front Behav Neurosci 1: 1.

21. Yang, M., Silverman, J. L. and Crawley, J. N. (2011). Automated three-chambered social approach task for mice. Curr Protoc Neurosci Chapter 8: Unit 826. 